\title{
Sparse reconstruction by convex relaxation: Fourier and Gaussian measurements
}

\author{
Mark Rudelson \\ Department of Mathematics \\ University of Missouri, Columbia \\ Columbia, Missouri 65211 \\ Email: rudelson@math.missouri.edu
}

\author{
Roman Vershynin \\ Department of Mathematics \\ University of California, Davis \\ Davis, California 95616 \\ Email: vershynin@math.ucdavis.edu
}

\begin{abstract}
This paper proves best known guarantees for exact reconstruction of a sparse signal $f$ from few non-adaptive universal linear measurements. We consider Fourier measurements (random sample of frequencies of $f$ ) and random Gaussian measurements. The method for reconstruction that has recently gained momentum in the Sparse Approximation Theory is to relax this highly non-convex problem to a convex problem, and then solve it as a linear program. What are best guarantees for the reconstruction problem to be equivalent to its convex relaxation is an open question. Recent work shows that the number of measurements $k(r, n)$ needed to exactly reconstruct any $r$-sparse signal $f$ of length $n$ from its linear measurements with convex relaxation is usually $O(r$ polylog $(n))$. However, known guarantees involve huge constants, in spite of very good performance of the algorithms in practice. In attempt to reconcile theory with practice, we prove the first guarantees for universal measurements (i.e. which work for all sparse functions) with reasonable constants. For Gaussian measurements, $k(r, n) \lesssim$ $11.7 r[1.5+\log (n / r)]$, which is optimal up to constants. For Fourier measurements, we prove the best known bound $k(r, n)=$ $O\left(r \log (n) \cdot \log ^{2}(r) \log (r \log n)\right)$, which is optimal within the $\log \log n$ and $\log ^{3} r$ factors. Our arguments are based on the technique of Geometric Functional Analysis and Probability in Banach spaces.
\end{abstract}

\section{INTRODUCTION}

During the last two years, the Sparse Approximation Theory benefited from a rapid development of methods based on the Linear Programming. The idea was to relax a sparse recovery problem to a convex optimization problem. The convex problem can be further be rendered as a linear program, and analyzed with all available methods of Linear Programming.

Convex relaxation of sparse recovery problems can be traced back in its rudimentary form to mid-seventies; references to its early history can be found in [26]. With the development of fast methods of Linear Programming in the eighties, the idea of convex relaxation became truly promising. It was put forward most enthusiastically and successfully by Donoho and his collaborators since the late eighties, starting from the seminal paper [15] (see Theorem 8, attributed there to Logan, and Theorem 9). There is extensive work being carried out, both in theory and in practice, based on the convex relaxation [8], [14], [16], [17], [13], [19], [24], [25], [26], [11], [9], [10], [12], [2], [1], [4], [5], [23], [3], [6], [20].

To have theoretical guarantees for the convex relaxation method, one needs to show that the sparse approximation problem is equivalent to its convex relaxation. Proving this presents a mathematical challenge. Known theoretical guarantees work only for random measurements (e.g. random Gaussian and Fourier measurements). Even when there is a theoretical guarantee, it involves intractable or very large constants, far worse than in the observed practical performances.

In this paper, we substantially improve best known theoretical guarantees for random Gaussian and Fourier (and nonharmonic Fourier) measurements. For the first time, we are able to prove guarantees with reasonable constants (although only for Gaussian measurements). Our proofs are based on methods of Geometric Functional Analysis, Such methods were recently successfully used for related problems [23], [20]. As a result, our proofs are reasonably short (and hopefully, transparent).

In Section $\amalg$ we state the sparse reconstruction problem and describe the convex relaxation method. A guarantee of its correctness is a very general restricted isometry condition on the measurement ensemble, due to Candes and Tao ([5], see [3]). Under this condition, the reconstruction problem with respect to these measurements is equivalent to its convex relaxation. In Sections [III and IV we improve best known guarantees for the sparse reconstruction from random Fourier (and nonharmonic Fourier) measurements and Gaussian measurements (Theorem 3.1 and 4.1 respectively).

\section{The Sparse Reconstruction Problem and its CONVEX RELAXATION}

We want to reconstruct an unknown signal $f \in \mathbb{C}^{n}$ from linear measurements $\Phi f \in \mathbb{C}^{k}$, where $\Phi$ is some known $k \times n$ matrix, called the measurement matrix. In the interesting case $k<n$, the problem is underdetermined, and we are interested in the sparsest solution. We can state this as the optimization problem

$$
\text { minimize }\left\|f^{*}\right\|_{0} \text { subject to } \Phi f^{*}=\Phi f,
$$

where $\|f\|_{0}=|\operatorname{supp} f|$ is the number of nonzero coefficients of $f$. This problem is highly non-convex. So we will consider its convex relaxation:

$$
\text { minimize }\left\|f^{*}\right\|_{1} \text { subject to } \Phi f^{*}=\Phi f,
$$


where $\|f\|_{p}$ denotes the $\ell_{p}$ norm throughout this paper, $\left(\sum_{i=1}^{n}\left|f_{i}\right|^{p}\right)^{1 / p}$. Problem (2) can be classically reformulated as the linear program

$$
\text { minimize } \sum_{i=1}^{n} t_{i} \text { subject to }-t \leq f^{*} \leq t, \Phi f^{*}=\Phi f,
$$

which can be efficiently solved using general or special methods of Linear Programming. Then the main question is:

Under what conditions on $\Phi$ are problems (1) and (2) equivalent?

In this paper, we will be interested in the exact reconstruction, i.e. we expect that the solutions to (1) and (2) are equal to each other and to $f$. Results for approximate reconstruction can be derived as consequences, see [4].

For exact reconstruction to be possible at all, one has to assume that the signal $f$ is $r$-sparse, that is $\operatorname{supp}(f) \leq r$, and that the number of measurements $k=k(r, n)$ has to be at least twice the sparsity $r$. Our goal will be to find sufficient conditions (guarantees) for the exact reconstruction. The number of measurements $k(r, n)$ should be kept as small as possible. Intuitively, the number of measurements should be of the order of $r$, which is the 'true' dimension of $f$, rather than the nominal dimension $n$.

Various results that appeared over the last two years demonstrate that many natural measurement matrices $\Phi$ yield exact reconstruction, with the number of measurements $k(r, n)=$ $O(r \cdot \operatorname{polylog}(n))$, see [2], [4], [5], [23]. In Sections III] and IV we improve best known estimates on $k$ for Fourier (and, more generally, nonharmonic Fourier) and Gaussian matrices respectively.

A general sufficient condition for exact reconstruction is the restricted isometry condition on $\Phi$, due to Candes and Tao ([5], see [3]). It roughly says that the matrix $\Phi$ acts as an almost isometry on all $O(r)$-sparse vectors. Precisely, we define the restricted isometry constant $\delta_{r}$ to be the smallest positive number such that the inequality

$$
C\left(1-\delta_{r}\right)\|x\|_{2}^{2} \leq\left\|\Phi_{T} x\right\|_{2}^{2} \leq C\left(1+\delta_{r}\right)\|x\|_{2}^{2}
$$

holds for some number $C>0$ and for all $x$ and all subsets $T \subset\{1, \ldots, n\}$ of size $|T| \leq r$, where $\Phi_{T}$ denotes the $k \times|T|$ matrix that consists of the columns of $\Phi$ indexed by $T$. The following theorem is due to Candes and Tao ([5], see [3]).

Theorem 2.1 (Restricted Isometry Condition): Let $\Phi$ be a measurement matrix whose restricted isometry constant satisfies

$$
\delta_{3 r}+3 \delta_{4 r} \leq 2 .
$$

Let $f$ be an $r$-sparse signal. Then the solution to the linear program (2) is unique and is equal to $f$.

This theorem says that under the restricted isometry condition (4) on the measurement matrix $\Phi$, the reconstruction problem (1) is equivalent to its convex relaxation (2) for all $r$-sparse functions $f$.

A problem with the use of Theorem 2.1 is that the restricted isometry condition (4) is usually difficult to check. Indeed, the number of sets $T$ involved in this condition is exponential in $r$. As a result, no explicit construction of a measurement matrix is presently known that obeys the restricted isometry condition (4). All known constructions of measurement matrices are randomized.

\section{RECONSTRUCTION FROM FOURIER MEASUREMENTS}

Our goal will be to reconstruct an $r$-sparse signal $f \in \mathbb{C}^{n}$ from its discrete Fourier transform evaluated at $k=k(r, n)$ points. These points will be chosen at random and uniformly in $\{0, \ldots, n-1\}$, forming a set $\Omega$.

The Discrete Fourier transform $\hat{f}=\Psi f$ is defined by the DFT matrix $\Psi$ with entries

$$
\Psi_{\omega, t}=\frac{1}{\sqrt{n}} \exp (-i 2 \pi \omega t / n), \quad \omega, t \in\{0, \ldots, n-1\} .
$$

So, our measurement matrix $\Phi$ is the submatrix of $\Psi$ consisting of random rows (with indices in $\Omega$ ). To be able to apply Theorem 2.1 it is enough to check that the restricted isometry condition (4) holds for the random matrix $\Phi$ with high probability. The problem is - what is the smallest number of rows $k(r, n)$ of $\Phi$ for which this holds? With that number, Theorem 2.1 immediately implies the following reconstruction theorem for Fourier measurements:

Theorem 3.1 (Reconstruction from Fourier measurements): A random set $\Omega \in\{0, \ldots, n-1\}$ of size $k(r, n)$ satisfies the following with high probability. Let $f$ be an $r$-sparse signal in $\mathbb{C}^{n}$. Then $f$ can be exactly reconstructed from the values of its Fourier transform on $\Omega$ as a solution to the linear program

$$
\text { minimize }\left\|f^{*}\right\|_{1} \text { subject to } \hat{f}^{*}(\omega)=\hat{f}(\omega), \quad \omega \in \Omega \text {. }
$$

The central remaining problem, what is the smallest value of $k(r, n)$, is still open. The best known estimate is due to Candes and Tao [4]:

$$
k(r, n)=O\left(r \log ^{6} n\right) .
$$

The conjectured optimal estimate would be $O(r \log n)$, which is known to hold for nonuniveral measuremets, i.e. for one sparse signal $f$ and for a random set $\Omega$ [2].

In this paper, we improve on the best known bound (5):

Theorem 3.2 (Sample size): Theorem 3.1 holds with

$$
k(r, n)=O\left(r \log (n) \cdot \log ^{2}(r) \log (r \log n)\right) .
$$

The dependence on $n$ is thus optimal within the $\log \log n$ factor and the dependence on $r$ is optimal within the $\log ^{3} r$ factor. So, our estimate is especially good for small $r$, but our estimate always yields $k(r, n)=O\left(r \log ^{4} n\right)$.

Remark 3.3: Our results hold for transforms more general than the discrete Fourier transform. One can replace the DFT matrix $\Psi$ by any orthogonal matrix with entries of magnitude $O(1 / \sqrt{n})$. Theorems 3.1 and 3.2 hold for any such matrix.

In the remainder of this section, we prove Theorem 3.2 Let $\Omega$ be a random subset of $\{0, \ldots, n\}$ of size $k$. Recall that the 
measurement matrix $\Phi$ that consists of the rows of $\Psi$ whose indices are in $\Omega$ ). In view of Theorem 3 , it suffices to prove that the restricted isometry constant $\delta_{r}$ of $\Phi$ satisfies

$$
\mathbb{E} \delta_{r} \leq \varepsilon
$$

whenever

$$
k \geq C\left(\frac{r \log n}{\varepsilon^{2}}\right) \log \left(\frac{r \log n}{\varepsilon^{2}}\right) \log ^{2} r,
$$

where $\varepsilon>0$ is arbitrary, and $C$ is some absolute constant.

Let $y_{1}, \ldots, y_{k}$ denote the rows of the matrix $\Psi$. Dualizing (3) we see that (6) is equivalent to the following inequality:

$$
\mathbb{E} \sup _{|T| \leq r}\left\|i d_{\mathbb{C}^{T}}-C^{\prime} \sum_{i \in \Omega} y_{i}^{T} \otimes y_{i}^{T}\right\| \leq \varepsilon
$$

with $C^{\prime}=1 / \sqrt{C}$. Here and thereafter, for vectors $x, y \in$ $\mathbb{C}^{n}$ the tensor $x \otimes y$ is the rank-one linear operator given by $(x \otimes y)(z)=\langle x, y\rangle z$, where $\langle\cdot\rangle$ is the canonical inner product on $\mathbb{C}^{n}$. The notation $x^{T}$ stands for the restriction of a vector $x$ on its coordinates in the set $T$. The operator $i d_{\mathbb{C}^{T}}$ in 8 is the identity on $\mathbb{C}^{T}$, and the norm is the operator norm for operators on $\ell_{2}^{T}$.

The orthogonality of $\Psi$ can be expressed as $i d_{\mathbb{C}^{n}}=$ $\sum_{i=0}^{n-1} y_{i} \otimes y_{i}$. We shall re-normalize the vectors $y_{i}$, letting $x_{i}=\sqrt{n} y_{i-1}$. Now we have $\left\|x_{i}\right\|_{\infty}=O(1)$ for all $i$. The proof has now reduced to the following probabilistic statement, which we interpret as a law of large numbers for random operators.

Theorem 3.4 (Uniform Operator Law of Large Numbers): Let $x_{1}, \ldots, x_{n}$ be vectors in $\mathbb{C}^{n}$ with uniformly bounded entries: $\left\|x_{i}\right\|_{\infty} \leq K$ for all $i$. Assume that $i d_{\mathbb{C}^{n}}=\frac{1}{n} \sum_{i=1}^{n} x_{i} \otimes x_{i}$. Let $\Omega$ be a random subset of $\{1, \ldots, n\}$ of size $k$. Then

$$
\mathbb{E} \sup _{|T| \leq r}\left\|i d_{\mathbb{C}^{T}}-\frac{1}{k} \sum_{i \in \Omega} x_{i}^{T} \otimes x_{i}^{T}\right\| \leq \varepsilon
$$

provided $k$ satisfies (7) (with constant $C$ that may depend on $K)$.

Theorem 3.4 is proved by the techniques developed in Probability in Banach spaces. The general roadmap is similar ton [21], [22]. We first observe that

$$
\mathbb{E} \frac{1}{k} \sum_{i \in \Omega} x_{i}^{T} \otimes x_{i}^{T}=\frac{1}{n} \sum_{i=1}^{n} x_{i}^{T} \otimes x_{i}^{T}=i d_{\mathbb{C}^{n}},
$$

so the random operator whose norm we estimate in (8) has mean zero. Then the standard symmetrization (see [27] Lemma 6.3) implies that the left-hand side of (8) does not exceed

$$
2 \mathbb{E} \sup _{|T| \leq r}\left\|\frac{1}{k} \sum_{i \in \Omega} \varepsilon_{i} x_{i}^{T} \otimes x_{i}^{T}\right\|
$$

where $\left(\varepsilon_{i}\right)$ are independent symmetric $\{-1,1\}$-valued random variables; also (jointly) independent of $\Omega$. Then the conclusion of Theorem 3.4 will be easily deduced from the following lemma.
Lemma 3.5: Let $x_{1}, \ldots, x_{k}, k \leq n$, be vectors in $\mathbb{C}^{n}$ with uniformly bounded entries, $\left\|x_{i}\right\|_{\infty} \leq K$ for all $i$. Then

$$
\mathbb{E} \sup _{|T| \leq r}\left\|\sum_{i=1}^{k} \varepsilon_{i} x_{i}^{T} \otimes x_{i}^{T}\right\| \leq k_{1} \sup _{|T| \leq r}\left\|\sum_{i=1}^{k} x_{i}^{T} \otimes x_{i}^{T}\right\|^{\frac{1}{2}}
$$

where $k_{1} \leq C_{1}(K) \sqrt{r} \log (r) \sqrt{\log n} \sqrt{\log k}$.

Let us show how Lemma 3.5 implies Theorem 3.4 We first condition on a choice of $\Omega$ and apply Lemma 3.5 for $x_{i}, i \in \Omega$. Then we take the expectation with respect to $\Omega$. We then use the a consequence of Hölder inequality, $\mathbb{E}\left(|X|^{\frac{1}{2}}\right) \leq(\mathbb{E}|X|)^{\frac{1}{2}}$ and the triangle inequality. Let us denote the left hand side of (8) by E. We obtain:

$$
E \leq \frac{2 k_{1}}{\sqrt{k}} \mathbb{E} \sup _{|T| \leq n}\left\|\frac{1}{k} \sum_{i \in \Omega} x_{i}^{T} \otimes x_{i}^{T}\right\|^{\frac{1}{2}} \leq \frac{2 k_{1}}{\sqrt{k}}(E+1)^{\frac{1}{2}} .
$$

It follows that $E \leq C_{2} \frac{2 k_{1}}{\sqrt{k}}$, provided that $\frac{2 k_{1}}{\sqrt{k}}=O(1)$. Theorem 3.4 now follows from our choice of $k=k(r, n)$.

Hence it is only left to prove Lemma 3.5 Throughout the proof, $B_{p}^{n}$ and $B_{p}^{T}$ denote the unit ball of the norm $\|\cdot\|_{p}$ on $\mathbb{C}^{n}$. To this end, we first replace Bernoulli r.v.'s $\varepsilon_{i}$ by standard independent normal random variables $g_{i}$, using a comparison principle (inequality (4.8) in [27]). Then our problem becomes to bound the Gaussian process, indexed by the union of the unit Euclidean balls $B_{2}^{T}$ in $\mathbb{C}^{T}$ for all subsets $I$ of $\{1, \ldots, n\}$ of size at most $r$. We apply Dudley's inequality (Theorem 11.17 in [27]), which is a general upper bound on Gaussian processes. Let us denote the left hand side of (8) by $E_{1}$. We obtain:

$$
\begin{aligned}
E_{1} & \leq C_{3} \mathbb{E} \sup _{|T| \leq r}\left\|\sum_{i=1}^{k} g_{i} x_{i}^{T} \otimes x_{i}^{T}\right\| \\
& =C_{3} \mathbb{E} \sup _{\substack{|T| \leq r \\
x \in B_{2}^{T}}}\left|\sum_{i=1}^{k} g_{i}\left\langle x_{i}, x\right\rangle^{2}\right| \\
& \leq C_{4} \int_{0}^{\infty} \log ^{1 / 2} N\left(\cup_{|T| \leq r} B_{2}^{T}, \delta, u\right) d u,
\end{aligned}
$$

where $N(Z, \delta, u)$ denotes the minimal number of balls of radius $u$ in metric $\delta$ centered in points of $Z$, needed to cover the set $Z$. The metric $\delta$ in Dudley's inequality is defined by the Gaussian process, and in our case it is

$$
\begin{aligned}
\delta(x, y) & =\left[\sum_{i=1}^{M}\left(\left\langle x_{i}, x\right\rangle^{2}-\left\langle x_{i}, y\right\rangle^{2}\right)^{2}\right]^{\frac{1}{2}} \\
& \leq\left[\sum_{i=1}^{k}\left(\left\langle x_{i}, x\right\rangle+\left\langle x_{i}, y\right\rangle\right)^{2}\right]^{\frac{1}{2}} \max _{i \leq k}\left|\left\langle x_{i}, x-y\right\rangle\right| \\
& \leq 2 \max _{|T| \leq r}\left[\sum_{z \in B_{2}^{T}}^{k}\left\langle x_{i}, z\right\rangle^{2}\right]^{\frac{1}{2}} \max _{i \leq k}\left|\left\langle x_{i}, x-y\right\rangle\right| \\
& =2 R \max _{i \leq k}\left|\left\langle x_{i}, x-y\right\rangle\right|,
\end{aligned}
$$


where

$$
R:=\sup _{|T| \leq r}\left\|\sum_{i=1}^{k} x_{i}^{T} \otimes x_{i}^{T}\right\|^{\frac{1}{2}}
$$

Hence

$$
E_{1} \leq C_{5} R \sqrt{r} \int_{0}^{\infty} \log ^{1 / 2} N\left(\frac{1}{\sqrt{r}} D_{2}^{r, n},\|\cdot\|_{X}, u\right) d u .
$$

Here

$$
D_{p}^{r, n}=\bigcup_{|T| \leq r} B_{p}^{T}, \quad\|x\|_{X}=\max _{i \leq k}\left|\left\langle x_{i}, x\right\rangle\right| .
$$

We will use containments

$$
\frac{1}{\sqrt{r}} D_{2}^{r, n} \subseteq D_{1}^{r, n} \subseteq K B_{X}, \quad D_{1}^{r, n} \subseteq B_{1}^{n},
$$

where $B_{X}$ denotes the unit ball of the norm $\|\cdot\|_{X}$. The second containment follows from the uniform boundedness of $\left(x_{i}\right)$. We can thus replace $\frac{1}{\sqrt{r}} D_{2}^{r, n}$ in (10) by $D_{1}^{r, n}$. Comparing (10) to the right hand side of (9) we see that, in order to complete the proof of Lemma 3.5, it suffices to show that

$\int_{0}^{K} \log ^{1 / 2} N\left(D_{1}^{r, n},\|\cdot\|_{X}, u\right) d u \leq C_{6} \log (r) \sqrt{\log n} \sqrt{\log k}$,

with $C_{6}=C_{6}(K)$. To this end, we will estimate the covering numbers in this integral in two different ways. For big $u$, we will just use the second containment in (11), which allows us to replace $D_{1}^{r, n}$ by $B_{1}^{n}$.

Lemma 3.6: Let $x_{1}, \ldots, x_{k}, k \leq n$, be vectors as in Lemma 3.5 Then for all $u>0$ we have

$$
N\left(B_{1}^{n},\|\cdot\|_{X}, u\right) \leq(2 n)^{m},
$$

where $m=C_{7} K^{2} \log (k) / u^{2}$.

Proof: We use the empirical method of Maurey. Fix a vector $y \in B_{1}^{n}$. Define a random vector $Z \in \mathbb{R}^{n}$ that takes values $(0, \ldots, 0, \operatorname{sign}(y(i)), 0, \ldots, 0)$ with probability $|y(i)|$ each, $i=1, \ldots, n$ (all entries of that vector are zero except $i$-th). Here $\operatorname{sign}(z)=z /|z|$, whenever $z \neq 0$, and 0 otherwise. Note that $\mathbb{E} Z=y$. Let $Z_{1}, \ldots, Z_{m}$ be independent copies of $Z$. Using symmetrization as before, we see that

$$
E_{3}:=\mathbb{E}\left\|y-\frac{1}{m} \sum_{j=1}^{m} Z_{j}\right\|_{X} \leq \frac{2}{m} \mathbb{E}\left\|\sum_{j=1}^{m} \varepsilon_{j} Z_{j}\right\|_{X} .
$$

Now we condition on a choice of $\left(Z_{j}\right)$ and take the expectation with respect to random signs $\left(\varepsilon_{j}\right)$. Using comparison to Gaussian variables as before, we obtain

$$
\begin{aligned}
E_{4} & :=\mathbb{E}\left\|\sum_{j=1}^{m} \varepsilon_{j} Z_{j}\right\|_{X} \leq C_{7} \mathbb{E}\left\|\sum_{j=1}^{m} g_{j} Z_{j}\right\|_{X} \\
& =C_{7} \mathbb{E} \max _{i \leq k}\left|\sum_{j=1}^{m} g_{j}\left\langle Z_{j}, x_{i}\right\rangle\right| .
\end{aligned}
$$

For each $i, \gamma_{i}:=\sum_{j=1}^{m} g_{j}\left\langle Z_{j}, x_{i}\right\rangle$ is a Gaussian random variable with zero mean and with variance

$$
\sigma_{i}=\left(\sum_{j=1}^{m}\left|\left\langle Z_{j}, x_{i}\right\rangle\right|^{2}\right)^{1 / 2} \leq K \sqrt{m}
$$

since $\left|\left\langle Z_{j}, x_{i}\right\rangle\right| \leq\left\|x_{i}\right\|_{\infty} \leq K$. Using a simple bound on the maximum of Gaussian random variables (see (3.13) in [27]), we obtain

$$
E_{4} \leq C_{7} \mathbb{E} \max _{i \leq k}\left|\gamma_{i}\right| \leq C_{8} \sqrt{\log k} \max _{i \leq k} \sigma_{i} \leq C_{8} \sqrt{\log k} K \sqrt{m} .
$$

Taking the expectation with respect to $\left(Z_{j}\right)$ we obtain

$$
E_{3} \leq \frac{2}{m} \mathbb{E}\left(E_{4}\right) \leq \frac{2 C_{8} K \sqrt{\log k}}{\sqrt{m}} .
$$

With the choice of $m$ made in the statement of the lemma, we conclude that $E_{3} \leq u$. We have shown that for every $y \in B_{1}^{n}$, there exists a $z \in \mathbb{C}^{n}$ of the form $z=\frac{1}{m} \sum_{j=1}^{m} Z_{j}$ such that $\|y-z\|_{X} \leq u$. Each $Z_{j}$ takes $2 n$ values, so $z$ takes $(2 n)^{m}$ values. Hence $B_{1}^{n}$ can be covered by at $(2 n)^{m}$ balls of norm $\|\cdot\|_{X}$ of radius $u$. A standard argument shows that we can assume that these balls are centered in points of $B_{1}^{n}$. This completes the proof of Lemma 3.6

For small $u$, we will use a simple volumetric estimate. The diameter of $B_{1}^{r}$ considered as a set in $\mathbb{C}^{n}$ is at most $K$ with respect to the norm $\|\cdot\|_{X}$ (this was stated as the last containment in (11). It follows that $N\left(B_{1}^{r},\|\cdot\|, u\right) \leq$ $(1+2 K / u)^{r}$ for all $r>0$, see (5.7) in [Pi]. The set $D_{1}^{r, n}$ consists of $d(r, n)=\sum_{j=1}^{r}\left(\begin{array}{c}n \\ i\end{array}\right)$ balls of form $B_{1}^{T}$, thus

$$
N\left(D_{1}^{r, n},\|\cdot\|_{X}, u\right) \leq d(n, r)(1+2 K / u)^{r} .
$$

Now we combine the estimate of the covering number $N(u)=\log ^{1 / 2} N\left(D_{1}^{r, n},\|\cdot\|_{X}, u\right)$ of Lemma 3.6, and the volumetric estimate (13), to bound the integral in (12). Using Stirling's approximation, we see that $d(r, n) \leq\left(C_{9} n / r\right)^{r}$. Thus

$$
\begin{gathered}
N(u) \leq C_{10} \sqrt{r}[\sqrt{\log (n / r)}+\sqrt{\log (1+2 / u)}]=: N_{1}(u), \\
N(u) \leq \frac{C_{10}}{u} \sqrt{\log k} \sqrt{\log n}=: N_{2}(u),
\end{gathered}
$$

where $C_{10}=C_{10}(K)$. Then we bound the integral in (12) as

$$
\begin{aligned}
\int_{0}^{K} N(u) d u \leq & \int_{0}^{A} N_{1}(u) d u+\int_{A}^{K} N_{2}(u) d u \\
\leq & C_{11} A \sqrt{r}[\sqrt{\log (n / r)}+\log (1+2 / A)] \\
& +C_{11} \log (1 / A) \sqrt{\log k} \sqrt{\log n}
\end{aligned}
$$

where $C_{11}=C_{11}(K)$. Choosing $A=1 / \sqrt{r}$, we conclude that the integral in (12) is at most $\sqrt{\log (n / r)}+\log r+$ $\log (r) \sqrt{\log k} \sqrt{\log n}$. This proves (12), which completes the proof of Lemma 3.5 and thus of Theorems 3.4 and 3.2 


\section{RECONSTRUCTION FROM GAUSSIAN MEASUREMENTS}

Our goal will be to reconstruct an $r$-sparse signal $f \in \mathbb{R}^{n}$ from $k=k(r, n)$ Gaussian measurements. These are given by $\Phi f \in \mathbb{R}^{k}$, where $\Phi$ is a $k \times n$ random matrix ('Gaussian matrix' in the sequel), whose entries are independent $N(0,1)$ random variables. The reconstruction will be achieved by solving the linear program (2).

The problem again is to find the smallest number of measurements $k(r, n)$ for which, with high probability, we have an exact reconstruciton of every $r$-sparse signal $f$ from its measurements $\Phi f$ ? It has recently been shown in [5], [23], [3] that

$$
k(r, n)=O(r \log (n / r)),
$$

and was extended in [20] to sub-gaussian measurements. This is asymptotically optimal. However, the constant factor implicit in (14) has not been known; previous proofs of (14) yield unreasonably weak constants (of order 2,000 and higher). In fact, there has not been known any theoretical guarantees with reasonable constants for Linear Programming based reconstructions. So, there is presently a gap between theoretical guarantees and good practical performance of reconstruction (2) (see e.g. [3]). Here we shall prove a first practically reasonable guarantee of the form (14):

$$
\begin{array}{r}
k(r, n) \leq c_{1} r\left[c_{2}+\log (n / r)\right](1+o(1)), \\
c_{1}=6+4 \sqrt{2} \approx 11.66, \quad c_{2}=1.5 .
\end{array}
$$

Theorem 4.1 (Reconstruction from Gaussian measurements): $A k \times n$ Gaussian matrix $\Phi$ with $k>k(r, n)$ satisfies the following with probability

$$
1-3.5 \exp \left(-(\sqrt{k}-\sqrt{k(r, n)})^{2} / 18\right) .
$$

Let $f$ be an r-sparse signal in $\mathbb{R}^{n}$. Then $f$ can be exactly reconstructed from the measurements $\Phi f$ as a unique solution to the linear program (2).

Our proof of Theorem 4.1 is direct, we will not use the Restricted Isometry Theorem 2.1 The first part of this argument follows a general method of [20]. One interprets the exact reconstruction as the fact that the (random) kernel of $\Phi$ misses the cone generated by the (shifted) ball of $\ell_{1}$. Then one embeds the cone in a universal set $D$, which is easier to handle, and proves that the random subspace does not intersect $D$. However, to obtain good constants as in (15), we will need to (a) improve the constant of embedding into $D$ from [20], and (b) use Gordon's Escape Through the Mesh Theorem [18], which is tight in terms of constants. In Gordon's theorem, one measures the size of a set $S$ in $\mathbb{R}^{n}$ by its Gaussian width

$$
w(D)=\mathbb{E} \sup _{x \in S}\langle g, x\rangle,
$$

where $g$ is a random vector in $\mathbb{R}^{n}$ whose components are independent $N(0,1)$ random variables (Gaussian vector). The following is Gordon's theorem [18].
Theorem 4.2 (Escape Through the Mesh (Gordon)): Let $S$ be a subset of the unit Euclidean sphere $S^{n-1}$ in $\mathbb{R}^{n}$. Let $Y$ be a random $(n-k)$-dimensional subspace of $\mathbb{R}^{n}$, distributed uniformly in the Grassmanian with respect to the Haar measure. Assume that $w(S)>\sqrt{k}$. Then $Y \cap S=\emptyset$ with probability at least

$$
1-3.5 \exp \left(-(k / \sqrt{k+1}-w(S))^{2} / 18\right) .
$$

We will now prove Theorem 4.1 First note that the function $f$ is the unique solution of (2) if and only if 0 is the unique solution of the problem

minimize $\left\|f-g^{*}\right\|_{1}$ subject to $\Phi g^{*} \in \operatorname{Ker}(\Phi)=: Y$.

$Y$ is a $(n-k)$-dimensional subspace of $\mathbb{R}^{n}$. Due to the rotation invariance of the Gaussian random vectors, $Y$ is distributed uniformly in the Grassmanian $G_{n-k, n}$ of $(n-k)$-dimensional subspaces of $\mathbb{R}^{n}$, with respect to the Haar measure.

Now, 0 is the unique solution to (16) if and only if 0 is the unique metric projection of $f$ onto the subspace $Y$ in the norm $\|\cdot\|_{1}$. This in turn is equivalent to the fact that 0 is the unique contact point between the subspace $Y$ and the ball of the norm $\|\cdot\|_{1}$ centered at $f$ :

$$
\left(f+\|f\|_{1} B_{1}^{n}\right) \cap Y=\{0\} .
$$

(Recall that $B_{p}^{n}$ is the unit ball of the norm $\|\cdot\|_{p}$.) Let $\mathcal{C}_{f}$ be the cone in $\mathbb{R}^{n}$ generated by the set $f+\|f\|_{1} B_{1}^{n}$ (the cone of a set $A \in \mathbb{R}^{n}$ is defined as $\left\{t a \mid a \in A, t \in \mathbb{R}^{+}\right\}$). Then the statement that 17 holds for all $r$-sparse functions $f$ is clearly equivalent to

$$
\mathcal{C}_{f} \cap Y=\{0\} \text { for all } r \text {-sparse functions } f .
$$

We can represent the cone $\mathcal{C}_{f}$ as follows. Let

$$
T^{+}=\{i \mid f(i)>0\}, T^{-}=\{j \mid f(i)<0\}, T=T^{+} \cup T^{-} \text {. }
$$

Then

$$
\mathcal{C}_{f}=\left\{t \in \mathbb{R}^{n}\left|\sum_{i \in T^{-}} t(i)-\sum_{i \in T^{+}} t(i)+\sum_{i \in T^{c}}\right| t(i) \mid \leq 0\right\} .
$$

We will now bound the cone $\mathcal{C}_{f}$ by a universal set, which does not depend on $f$.

Lemma 4.3: Consider the spherical part of the cone, $K_{f}=$ $\mathcal{C}_{f} \cap S^{n-1}$. Then $K_{f} \subset(\sqrt{2}+1) D$, where

$$
D=\operatorname{conv}\left\{x \in S^{n-1}|| \operatorname{supp}(x) \mid \leq r\right\} .
$$

Proof: Fix a point $x \in \mathcal{C} \cap S^{n-1}$. We have

$$
\sum_{i \in T}|x(i)| \leq \sqrt{|I|} \leq \sqrt{r}, \quad \sum_{i \in T^{c}}|x(i)| \leq \sum_{i \in T}|x(i)| \leq \sqrt{r} \text {. }
$$

The norm $\|\cdot\|_{D}$ on $\mathbb{R}^{n}$ whose unit ball is $D$ can be computed as

$$
\|x\|_{D}=\sum_{l=1}^{L}\left(\sum_{i \in I_{l}}\left(x(i)^{*}\right)^{2}\right)^{1 / 2}
$$

where $L=\lceil n / r\rceil, I_{l}=\{r(l-1)+1, \ldots, r l\}$, for $l<L$, $I_{L}=\{r(L-1)+1, \ldots, n\}$, and $\left(x(i)^{*}\right)$ is a non-decreasing rearrangement of the sequence $(|x(i)|)$. 
Set $F=F(x)=\{i|| x(i) \mid \geq 1 / \sqrt{r}\}$. Since $x \in S^{n-1}$, we have $|F| \leq r$. Hence, for any $x \in K$ there exists a set $E=E(x) \subset\{1, \ldots, m\}$, which consists of $2 r$ elements and such that $E \supseteq F \cup I$. Therefore, $x$ can be represented as $x=x^{\prime}+x^{\prime \prime}$ so that $\operatorname{supp}\left(x^{\prime}\right) \subseteq E,\|x\|_{2} \leq 1, \operatorname{supp}\left(x^{\prime \prime}\right) \subseteq E^{c}$, $\left\|x^{\prime \prime}\right\|_{\infty} \leq 1 / \sqrt{r}$. Set

$$
V_{E}=B_{2}^{E} \times\left(\sqrt{r} B_{1}^{E^{c}} \cap \frac{1}{\sqrt{r}} B_{\infty}^{E^{c}}\right) .
$$

Then the above argument shows that $K_{f} \subset \bigcup_{|E|=2 r} V_{E}=: W$.

The maximum of $\|x\|_{D}$ over $x \in W$ is attained at the extreme points of the sets $V_{E}$, which have the form $x=x^{\prime}+$ $x^{\prime \prime}$, where $x^{\prime} \in S^{E}$, and $x^{\prime \prime}$ has coordinates 0 and $\pm 1 / \sqrt{r}$ with $r$ non-zero coordinates. Notice that since $\left|\operatorname{supp}\left(x^{\prime}\right)\right| \leq 2 r$, $\left\|x^{\prime}\right\|_{D} \leq \sqrt{2}\left\|x^{\prime}\right\|_{2}$. Thus, for any extreme point $x$ of $V_{E}$,

$$
\|x\|_{D} \leq\left\|x^{\prime}\right\|_{D}+\left\|x^{\prime \prime}\right\|_{D} \leq \sqrt{2}\left\|x^{\prime}\right\|_{2}+\left\|x^{\prime \prime}\right\|_{2} \leq \sqrt{2}+1 .
$$

The second inequality follows from $\operatorname{supp}\left(x^{\prime}\right) \leq 2 r$ and $\operatorname{supp}\left(x^{\prime \prime}\right)=r$. This completes the proof of the lemma.

To use Gordon's escape through the mesh theorem, we have to estimate the Gaussian width of $D$.

Lemma 4.4:

$$
w(D) \leq \sqrt{2 r \log \left(e^{3 / 2} n / r\right)}(1+o(1)) .
$$

Proof: By definition,

$$
w(D)=\sup _{|J|=r}\left(\sum_{i \in J}|g(i)|^{2}\right)^{1 / 2} .
$$

Let $p>1$ be a number to be chosen later. By Hölder's inequality, we have

$$
\begin{aligned}
w(D) & \leq \mathbb{E}\left(\sum_{|J|=r}\left(\sum_{i \in J}|g(i)|^{2}\right)^{p / 2}\right)^{1 / p} \\
& \leq\left(\begin{array}{c}
n \\
r
\end{array}\right)^{1 / p}\left(\mathbb{E}\left(\sum_{i=1}^{r}|g(i)|^{2}\right)^{p / 2}\right)^{1 / p} \\
& \leq\left(\frac{e n}{r}\right)^{r / p}\left(2^{p / 2} \cdot \frac{\Gamma(p / 2+r / 2)}{\Gamma(r / 2)}\right)^{1 / p} .
\end{aligned}
$$

By the Stirling's formula,

$$
2^{p / 2} \cdot \frac{\Gamma(p / 2+r / 2)}{\Gamma(r / 2)}=\left(1+\frac{p}{r}\right)^{\frac{r+1}{2}}\left(\frac{p+r}{e}\right)^{p / 2}(1+o(1)) .
$$

Therefore, $w(D) \leq\left(\frac{e n}{r}\right)^{r / p}\left(\frac{p+r}{e}\right)^{1 / 2}(1+o(1))$. Now set $p=2 r \log \left(\frac{e n}{r}\right)$. Then

$$
w(D) \leq(p+r)^{1 / 2}(1+o(1))=\sqrt{2 r \log \frac{e^{3 / 2} n}{r}}(1+o(1)) .
$$

To deduce (18) we define $S=\bigcup_{f} K_{f}$, where the union is over all $r$-sparse functions $f$. Then (18) is equivalent to

$$
S \cap Y=\emptyset .
$$

Lemma 4.3 implies that $S \subseteq(\sqrt{2}+1) D$. Then by Lemma 4.4

$$
w(S) \leq(\sqrt{2}+1) w(D)=(1-o(1)) \sqrt{k(r, n)} .
$$

Then (19) follows Gordon's Theorem 4.2 This completes the proof of Theorem 4.1

Acknowledgement. After this paper was announced, A.Pajor pointed out that Lemma 3.6 was proved by B.Carl in [7], see Prop.3 and below. We also thank Emmanuel Candes for important remarks.

\section{REFERENCES}

[1] E. Candes, J. Romberg, Quantitative Robust Uncertainty Principles and Optimally Sparse Decompositions, Foundations of Computational Mathematics, to appear

[2] E. Candes, J. Romberg, T. Tao, Robust Uncertainty Principles: Exact Signal Reconstruction from Highly Incomplete Frequency Information, preprint

[3] E. Candes, M. Rudelson, T. Tao, R. Vershynin, Error correction via Linear Programming, FOCS 2005 (46th Annual Symposium on Foundations of Computer Science), 295-308

[4] E. Candes, T. Tao, Near Optimal Signal Recovery From Random Projections: Universal Encoding Strategies?, preprint

[5] E. Candes, T. Tao, Decoding by Linear Programming, preprint

[6] E. Candes, T. Tao, The Dantzig selector: statistical estimation when $p$ is much larger than $n$ Annals of Statistics, to appear

[7] B. Carl, Inequalities of Bernstein-Jackson-type and the degree of compactness of operators in Banach spaces, Ann. Inst. Fourier (Grenoble) 35 (1985), 79-118

[8] S. Chen, D. Donoho, M. Saunders, Atomic decomposition by basis pursuit, SIAM J. Sci. Comput. 20 (1998), no. 1, 33-61; reprinted in: SIAM Rev. 43 (2001), no. 1, 129-159

[9] D. Donoho, For Most Large Underdetermined Systems of Linear Equations, the minimal $\ell_{1}$-norm solution is also the sparsest solution, preprint

[10] D. Donoho, For Most Large Underdetermined Systems of Linear Equations, the minimal l1-norm near-solution approximates the sparsest nearsolution, preprint

[11] D. Donoho, M. Elad, V. Temlyakov, Stable Recovery of Sparse Overcomplete Representations in the Presence of Noise, preprint

[12] D. Donoho, Y. Tsaig, Extensions of compresed sensing, preprint

[13] D. Donoho, M. Elad, Optimally sparse representation in general (nonorthogonal) dictionaries via ell 1 minimization, Proc. Natl. Acad. Sci. USA 100 (2003), 2197-2202

[14] D. Donoho, X. Huo, Uncertainty principles and ideal atomic decomposition, IEEE Trans. Inform. Theory 47 (2001), 2845-2862

[15] D. Donoho, P. Stark, Uncertainty principles and signal recovery, SIAM J. Appl. Math. 49 (1989), 906-931

[16] M. Elad, A. Bruckstein, A generalized uncertainty principle and sparse representation in pairs of bases, IEEE Trans. Inform. Theory 48 (2002), 2558-2567

[17] A. Feuer, A. Nemirovski, On sparse representation in pairs of bases, IEEE Trans. Inform. Theory 49 (2003), 1579-1581

[18] Y. Gordon, On Milman's inequality and random subspaces which escape through a mesh in $\mathbb{R}^{n}$, Geometric aspects of functional analysis, Isr Semin. 1986-87, Lect. Notes Math. 1317, 84-106 (1988)

[19] R. Gribonval, M. Nielsen, Sparse representations in unions of bases, IEEE Trans. Inform. Theory 49 (2003), 3320-3325.

[20] S. Mendelson, A. Pajor, N. Tomczak-Jaegermann, Reconstruction and subgaussian operators, preprint

[21] M. Rudelson, Approximate John's decompositions, Operator Theory: Advances and Applications 77(1995), 245-249.

[22] M. Rudelson, Random vectors in the isotropic position, J. of Functional Analysis 164 (1999), 60-72.

[23] M. Rudelson, R. Vershynin, Geometric approach to error correcting codes and reconstruction of signals, to appear in Int. Math. Res. Notices

[24] J. Tropp, Recovery of short, complex linear combinations via $\ell_{1}$ minimization, IEEE Trans. Inform. Theory, to appear

[25] J. Tropp, Greed is good: Algorithmic results for sparse approximation, IEEE Trans. Inform. Theory, Vol. 50, Num. 10, October 2004, pp. 22312242

[26] J. Tropp, Just relax: Convex programming methods for subset selection and sparse approximation, ICES Report 04-04, UT-Austin, February 2004.

[27] M. Ledoux and M. Talagrand, Probability in Banach spaces, Springer, 1991.

[Pi] G. Pisier, The volume of convex bodies and Banach space geometry, Cambridge Tracts in Mathematics 94, Cambridge University Press, 1989. 